\title{
Near-space slow SAR high-resolution wide swath and sustained imaging technology
}

\author{
Haiguang Yang*, Jianyu Yang, Zhongyu Li, Junjie Wu, Yulin Huang and Long Teng
}

\begin{abstract}
Sustained high-resolution imaging for wide swath area is getting emergence. However, conventional airborne and spaceborne SAR can hardly achieve them simultaneously. Near-space technology draws much attention in this aspect. Current researches of near-space SAR focus on fast platform which encounters contradiction between azimuth high resolution and range wide swath. To solve it and achieve sustained imaging, low-speed SAR is introduced in this article. Joint aperture technology is then proposed to make full use of the redundant pulse repetition frequency and obtain wide area imaging fast. Performance of the example system given at the end of the article verifies the effectiveness of the proposed near-space low-speed SAR high-resolution wide swath and sustained imaging concepts.
\end{abstract}

Keywords: Near-space, Low-speed, High-resolution, Wide swath, Sustained, Joint aperture technology

\section{Introduction}

High-resolution wide swath (HRWS) and sustained imaging is of great significance in many fields, like public security, disaster warning, etc. Conventional airborne SAR is capable of high-resolution narrow swath imaging while spaceborne on the contrary $[1,2]$. Also, it is impossible of sustained imaging for both of them due to revisiting frequency.

Near-space is recognized as the altitudes between 20 and $100 \mathrm{~km}[3,4]$. As Figure 1a shows, it is high for airplanes and too low for satellites. Near-space vehicles offer several advantages such as persistence, robust survivability, and cost efficiency. In addition, the velocities of near-space vehicles can range from nearly stationary to $1500 \mathrm{~m} / \mathrm{s}$. Because of the unique higher altitude and the velocity of near-space platforms, near-space SAR can achieve the capacities of HRWS and sustained imaging, simultaneously.

Current researches of near-space SAR focus on highspeed platforms [5-8]. However, it's inherent defect of contradiction between azimuth high resolution and rang wide swath cannot be solved by conventional technologies. Multichannel- or multiaperture-based techniques [2,5-11] are introduced to solve it. However, multiaperture in

\footnotetext{
* Correspondence: yanghaiguang@uestc.edu.cn

School of Electronic Engineering, University of Electronic Science and Technology of China, Chengdu 611731, People's Republic of China
}

platform drives whole system more complex and heavy. And also it has other channel matching problems.

In recent news, a new concept of low-speed platform is coming into sight. From the strategic point of view, low-speed could be sorted into two types: airship and balloon [12-14]. As Figure 1b shows, typical airship systems are the DARPA's high-altitude airship (HAA), Integrated Sensor Is Structure (ISIS) Unmanned HALE airship. Also the Blue Devil is developed for battle reconnaissance in Afghanistan. Meanwhile, balloon slowspeed system is also developed. Near-space diameter balloon with 400-ft. structure is on developing in Spain and it could float $36 \mathrm{~km}$ high to take a glimpse of the curvature of earth. Even the India's army took hand on it and developed the Akashdeep Aerostat.

So in this article, we present the design of a near-space low-speed SAR (NSLS-SAR), and its imaging strategy. In general, the NSLS-SAR adopts balloon and airship to balance its own gravity, so energy is saved for devices. Sunlight in near-space could then replenish energy consumption and ensure the sustained imaging.

Most importantly, low-speed brings redundant samplings in spatial domain which can hardly be obtained in high-speed system. Redundant sampling then solves the contradiction between azimuth high-resolution and range wide swath while keeps conventional radar system. However, redundant sampling in spatial domain brings time

\section{实}

(c) 2013 Yang et al.; licensee Springer. This is an Open Access article distributed under the terms of the Creative Commons Attribution License (http://creativecommons.org/licenses/by/2.0), which permits unrestricted use, distribution, and reproduction in any medium, provided the original work is properly cited. 


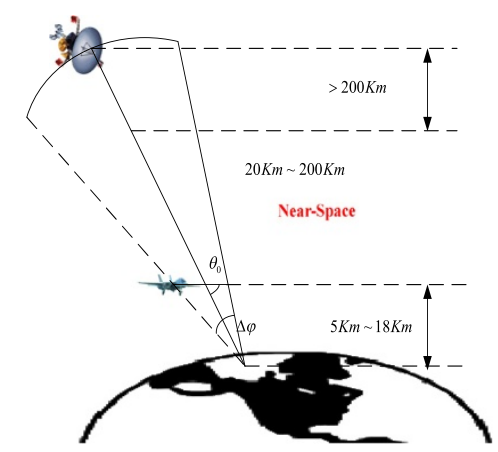

(a)

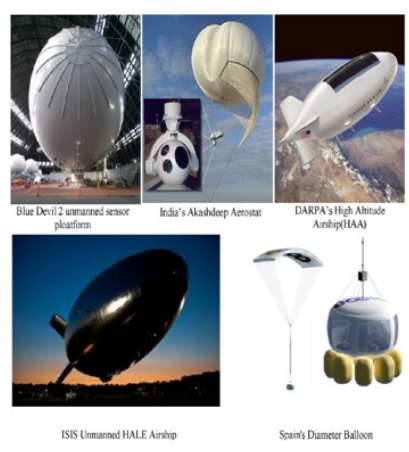

(b)

Figure 1 Space division and several typical near-space low-speed platforms. (a) Space division. (b) Near-space low-speed platforms.

expansion and takes much longer time for the same azimuth size. This is called time space exchange. Meanwhile, in order to overcome the problem of long synthetic time, new concept of joint aperture technology (JAT) is firstly proposed for fast imaging in this article.

The remainder of this article is organized as follows. Section 2 introduces capability of near-space in highresolution, wide swath imaging and figures out current researches as well as challenges encountered. Section 3 proposes low-speed SAR and discusses the idea of time space exchange, new issue of long synthetic time. JAT that solves this new issue is then proposed in Section 4. Details of JAT and following assembling process also described. The performance of an example system verifies the effectiveness of the proposed concepts in Section 5. Finally, Section 6 concludes the article.

\section{Current near-space SAR and problems}

Based on the SAR resolution theory, a high azimuth resolution requires a short antenna to illuminate a long synthetic aperture, viz. it will result in a wide Doppler bandwidth. To satisfy the Nyquist sampling criterion, this azimuth bandwidth must be sampled at a high pulse repetition frequency (PRF). However, a high PRF means a smaller swath width. The relation between the maximum imaging swath on ground $W_{s}$ and the required PRF can be expressed as

$$
W_{s} \leq \frac{c}{2 \cdot \mathrm{PRF} \cdot \sin \theta_{0}}
$$

where $c$ and $\theta_{0}$ denote the speed of light and the incidence angle, respectively. That is to say a low PRF is favorable to unambiguously image a wide swath on the ground.

And based on the pulse compression theorem, the azimuth resolution is

$$
\rho_{a}=\frac{v}{B_{a}} \geq \frac{v}{\mathrm{PRF}}
$$

where $v$ is the speed of the platform, and $B_{a}$ denotes the doppler bandwidth.

Substituting Equation (2) into (1), we can get the following restriction

$$
\frac{W_{s}}{\rho_{a}} \leq \frac{c}{2 \cdot v \cdot \sin \theta_{0}}
$$

Generally speaking, $c / v$ is nearly constant at 20,000 for spaceborne SARs and typically in range of 3,00,000750,000 for airborne SARs. In contrast, near-space platforms can fly at a speed ranging from nearly stationary to $1500 \mathrm{~m} / \mathrm{s}$, then the corresponding $c / v$ will be greater than 100,000. Thus, compared with spaceborne and airborne SARs, near-space SAR can provide a more flexible choice between azimuth resolution and swath width for satisfy the high-resolution and wide-swath imaging.

Also, Equation (2) can also be reformed into the basic minimum antenna area $\left(A_{\text {antenna }}\right)$ constrain

$$
A_{\text {antenna }} \geq \frac{4 v \lambda R_{c} \cdot \tan \left(\theta_{0}\right)}{c}
$$

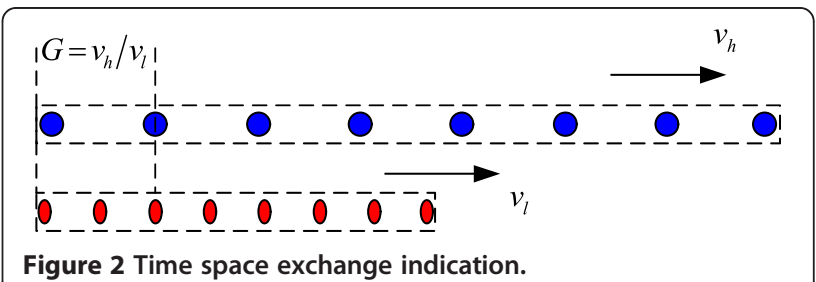




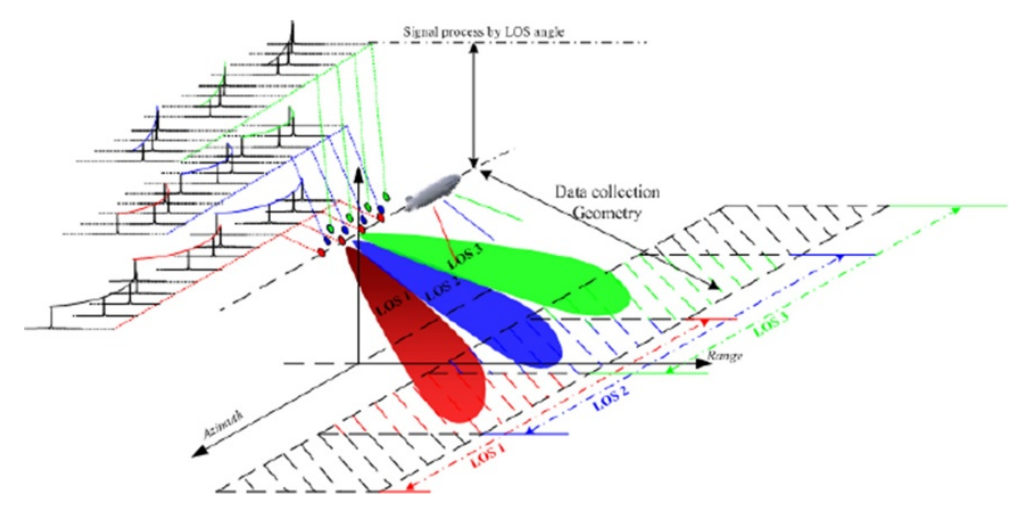

Figure 3 The geometry configuration of JAT.

where $\lambda$ is the radar wavelength and $R_{c}$ is the slant range from the radar to the midswath.

In reported materials, researches of near-space SAR focus on high-speed platform [5-8]. From Equation (4) we can get that, with regard to the high-speed platforms, the minimum antenna area of $A_{\text {antenna }}$ will relatively be large. However, in order to achieve the HRWS imaging, the antenna area should be small. So, as to alleviate the requirements imposed on the minimum antenna area for the high-speed platform, several multichannel- or multiaperture-based techniques, such as displaced phase center antenna technique [15], digital beamforming technique [5-8], viz., have been proposed.

Nevertheless, multichannel- or multiaperture on receiver greatly increases the complexity of the whole system both in hardware and software, such as requiring additional complex signal process before imaging process to extract expected signal. This not only complicates the system design, but also brings weight burden for platform.

To address these problems for high-speed platforms, we present the design of NSLS-SAR, and its imaging strategy in the following sections.

\section{NSLS-SAR scheme}

Low-speed platforms operate at speed of $0-30 \mathrm{~m} / \mathrm{s}$ which is dozens of times smaller than high-speed SAR. Because of this special speed, from Equation (4) we can get that
NSLS-SAR could adopt energy-efficient platform and solve restrictions above while keeps conventional radar structure. For example, assume the speed of the platform is $30 \mathrm{~m} / \mathrm{s}$, the azimuth resolution is $0.5 \mathrm{~m}$, then the Doppler bandwidth is $60 \mathrm{~Hz}$, so the PRF should be larger than $60 \mathrm{~Hz}$. And also we assume the large rang width is $200 \mathrm{~km}$, then we can get the PRF should be smaller than $750 \mathrm{~Hz}$. We can see that the two restrictions are not contradictory. So, NSLS-SAR can avoid the problems for highspeed platforms. Meanwhile, because of the low-speed, it can also achieve the sustained imaging as explained in the following sections.

\section{Sustained imaging}

Low-speed SAR uses airship or balloon as its platform while high-speed SAR uses vehicles. Vehicles are born with defect of short air-staying time while airship and balloon are perfect of suspension in the air. Moreover, sunlight is plenty in near-space which enables energy replenishing. Wind direction is stable for sustained imaging. In this way, low-speed SAR could operate months and even years in certain area. So, NSLS-SAR can achieve the sustained imaging.

\section{Time space exchange}

Conventional SAR encounters contradiction between wide swath and azimuth resolution because of PRF limitation.

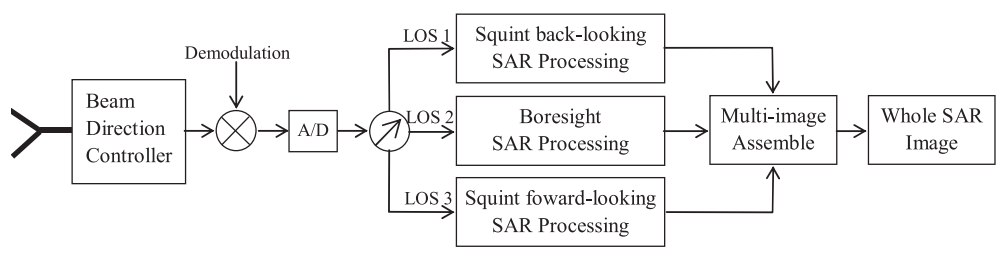

Figure 4 The system structure of NSLS-SAR. 


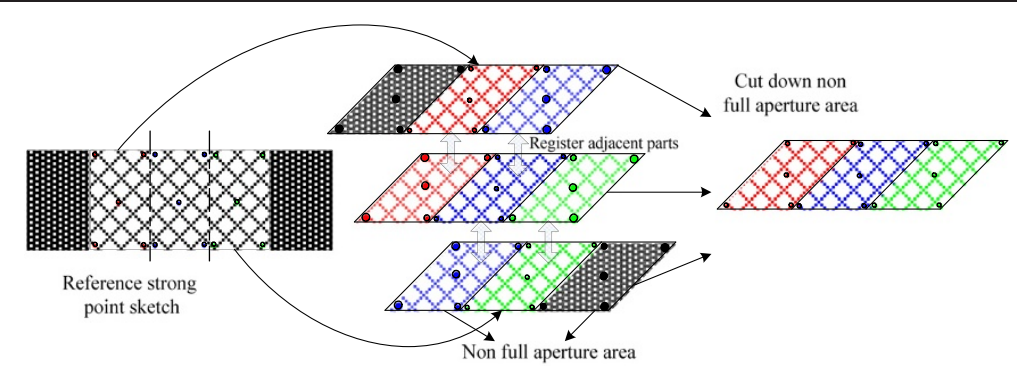

Figure 5 Multi-image assembling process.

For low-speed SAR, however, possess flexible vehicle speed that ranges from zero to $30 \mathrm{~m} / \mathrm{s}$ typically. This distinctive feature allows couples times of PRF extension. When the platform speed cuts down to two-thirds as before, the azimuth bandwidth for the same resolution also cut down to two-thirds. Finally, PRF requirement cut down the same percent.

We define a factor $G$ to represent the ratio of reference velocity to actual velocity. Low-speed SAR holds a $G$ value of over 50 with reference of high-speed SAR. Thus, allows over 50 times lower PRF requirement. In this case, conventional contradiction is out of existence and even brings a redundant characteristic in PRF.

However, the strategy of low-speed affects synthetic length and surveillance coverage the other way round. Take a conventional high-speed SAR as reference, lowspeed SAR holds flexible factor G. As Figure 2 shows, for the same synthetic time, low-speed covers $1 / G$ time synthetic length as fast one does.

This could be called as time space exchange. Redundant sampling in time domain is obtained at the cost of synthetic length in spatial domain. However, new issues short synthetic length means short surveillance coverage in azimuth direction which is greatly adverse to fast imaging and real-time monitoring.

Table 1 NSLS-SAR example system parameters

\begin{tabular}{cc}
\hline Parameters & Values \\
\hline Carrier frequency & $9.6 \mathrm{GHz}$ \\
\hline Platform velocity & $30 \mathrm{~m} / \mathrm{s}$ \\
\hline Platform altitude & $70 \mathrm{~km}$ \\
\hline Minimum slant range & $80 \mathrm{~km}$ \\
\hline Maximum slant range & $280 \mathrm{~km}$ \\
\hline Azimuth imaging boundary & -45 to $45 \mathrm{~km}$ \\
\hline Number of Joint aperture & 9 \\
\hline Pulse duration time & $10 \mathrm{\mu s}$ \\
\hline Pulse bandwidth & $200 \mathrm{MHz}$ \\
\hline Range sampling frequency & $240 \mathrm{MHz}$ \\
\hline
\end{tabular}

\section{JAT}

In general, PRF is several times larger than the azimuth bandwidth of NSLS-SAR, viz. the PRF is redundant. In order to deal with the long synthetic time caused by the low-speed, we will make full use of the redundant PRF and propose the JAT.

The main idea of JAT is mapping one aperture to various ground area to accomplish large azimuth area surveillance. In JAT, certain large area is divided into several parts with overlapping area between neighbors, and the azimuth width of the overlapping area is one synthetic aperture length, which will be used to assemble the multi-images. Radar periodically changes its line-of -sight (LOS) angle to illuminate each part of the area. Under this circumstance, multi-imaging areas share the same aperture during synthetic time and large imaging areas are generated fast. After imaging process, register adjacent part and assemble the whole image. We will discuss in more detail of the JAT in the following section.

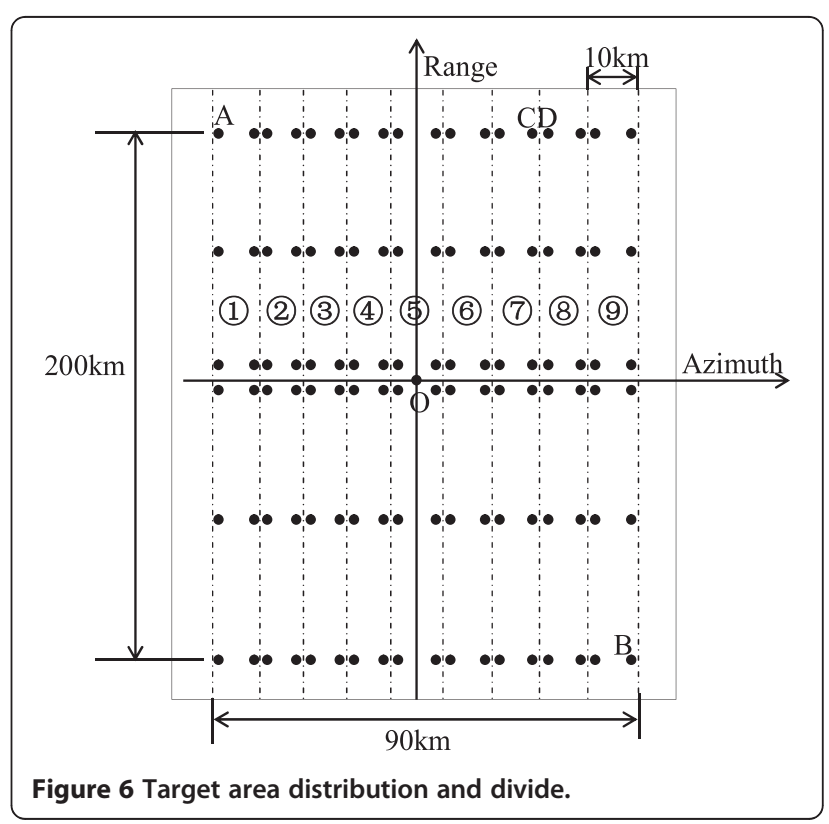




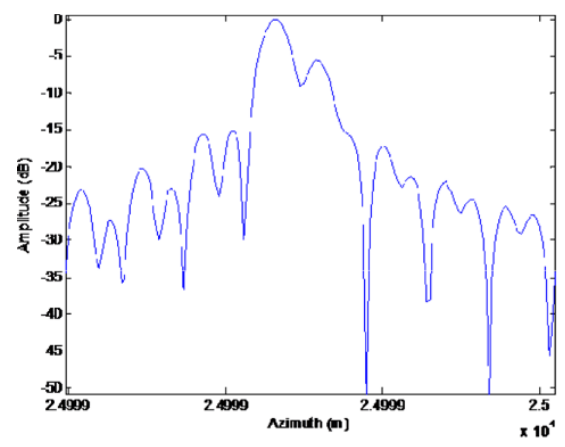

(a)

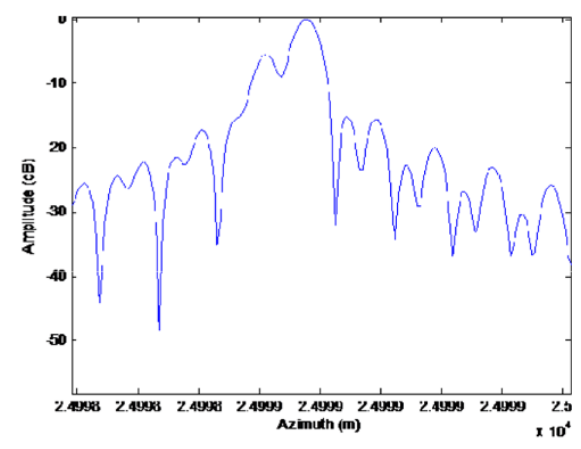

(b)

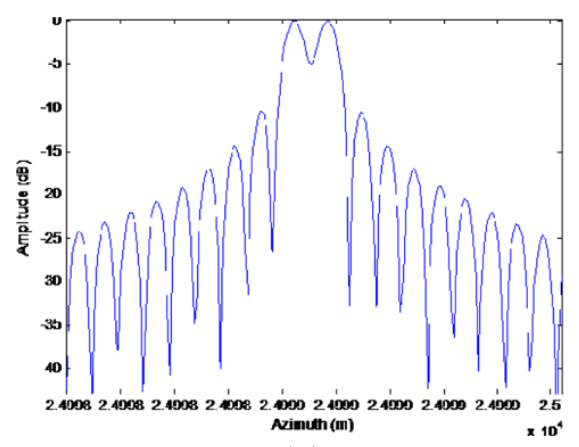

(c)

Figure 7 Azimuth profiles of targets C and D. (a) LOS 7. (b) LOS 8. (c) Assembled image.

\section{Target area divide}

As shown in Figure 3, radar periodical changes its LOS angle to cover large area. The cycle time equals to a pulse repetition interval (PRI). For each LOS angle, beam form illuminates a unique area. The number of the LOS (viz. $N$ ) should be satisfy the following restriction

$$
N \leq \operatorname{round}\left(\frac{\mathrm{PRF}}{B_{a}}\right)
$$

where round(.) represents rounding operation. Then the required time of the whole area imaging will cut down to $1 / N$ of the traditional stripmap mode.

Take a three LOS angles SAR as an example. At first PRI, LOS 1 illuminates the red area, then at the second PRI, LOS 2 illuminates blue area, and at the third PRI, LOS 3 illuminates green one. In the second cycle, viz. at the fourth PRI, the LOS of the radar turns back to LOS 1, etc. Each part closes to each other with some overlapping region, and the azimuth width of the overlapping area is one synthetic aperture length, which will be used to assemble the adjacent multi-images. The example system structure of NSLS-SAR is shown in Figure 4.
Let the transmitted signal be a chirp

$$
S(\tau)=\operatorname{rect}\left[\frac{\tau}{T_{r}}\right] \exp \left(-j \pi K_{r} \tau^{2}+j 2 \pi f_{0} \tau\right)
$$

where rect[.] is the fast-time envelope, $T_{r}$ represents the pulse duration time, $\tau$ is the fast time variable, $K_{r}$ is the FM rate, and $f_{r}$ is the carrier frequency.

After demodulation to baseband, the echo received by LOS 1 can be written as

$$
\begin{aligned}
S_{\mathrm{LOS} 1}\left(\tau, t_{1}\right)= & \iint_{\mathrm{LOS} 1} \sigma(x, y) \operatorname{rect}\left[\frac{\tau-\tau_{d}\left(t_{1} ; x, y\right)}{T_{r}}\right] \omega_{a}\left[\frac{t_{1}-t_{d}(y)}{T_{a}}\right] \\
& \times \exp \left\{j \pi K_{r}\left[\tau-\frac{2 R\left(t_{1} ; x, y\right)}{c}\right]\right\} \\
& \times \exp \left\{-j 4 \pi f_{0} R\left(t_{1} ; x, y\right) / c\right\} d x d y
\end{aligned}
$$

where $t_{1} \in$ PRI [ $14.710 \cdots$ ] is the azimuth time variable, and $\tau_{d}\left(t_{1} ; x, y\right)=\frac{2 R\left(t_{1} ; x, y\right)}{c}$. The echo of LOS 1 is squint back-looking mode, and then it will be processed by the squint back-looking SAR processing method. 


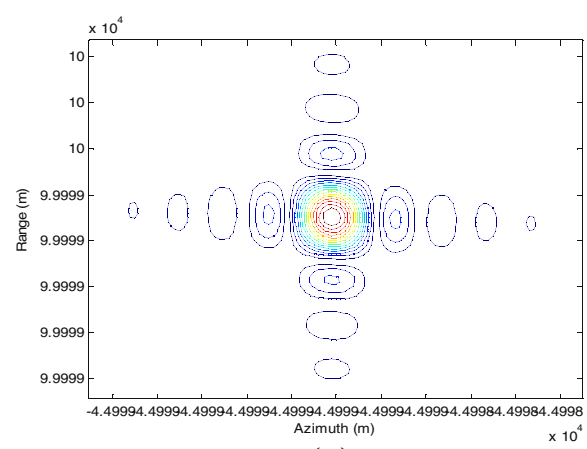

(a)

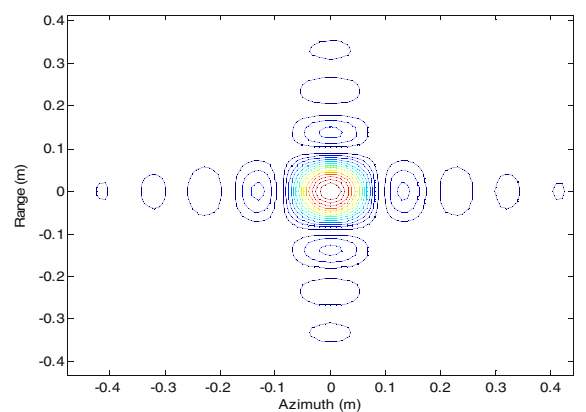

(b)

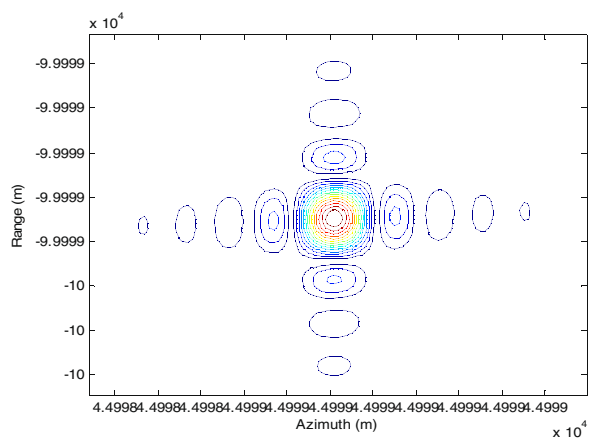

(c)

Figure 8 Focusing results by the NSLS-SAR imaging technology. (a) Target A. (b) Target O. (c) Target B.

The echo of LOS 2 is

$$
\begin{aligned}
S_{\mathrm{LOS} 2}\left(\tau, t_{2}\right)= & \iint_{\mathrm{LOS} 2} \sigma(x, y) \operatorname{rect}\left[\frac{\tau-\tau_{d}\left(t_{2} ; x, y\right)}{T_{r}}\right] \omega_{a}\left[\frac{t_{2}-t_{d}(y)}{T_{a}}\right] \\
& \times \exp \left\{j \pi K_{r}\left[\tau-\frac{2 R\left(t_{2} ; x, y\right)}{c}\right]\right\} \\
& \times \exp \left\{-j 4 \pi f_{0} R\left(t_{2} ; x, y\right) / c\right\} d x d y
\end{aligned}
$$

where $t_{2} \in P R I\left[\begin{array}{lllll}2 & 5 & 8 & 11 \cdots\end{array}\right]$, and $\tau_{d}\left(t_{2} ; x, y\right)=\frac{2 R\left(t_{2} ; x, y\right)}{c}$. The echo of LOS 2 is boresight mode, and then it will be processed by the boresight SAR processing method.

The echo of LOS 3 is

$$
\begin{aligned}
S_{\mathrm{LOS} 3}\left(\tau, t_{3}\right)= & \int_{\operatorname{LOS} 3} \sigma(x, y) \operatorname{rect}\left[\frac{\tau-\tau_{d}\left(t_{3} ; x, y\right)}{T_{r}}\right] \omega_{a}\left[\frac{t_{3}-t_{d}(y)}{T_{a}}\right] \\
& \times \exp \left\{j \pi K_{r}\left[\tau-\frac{2 R\left(t_{3} ; x, y\right)}{c}\right]\right\} \\
& \times \exp \left\{-j 4 \pi f_{0} R\left(t_{3} ; x, y\right) / c\right\} d x d y
\end{aligned}
$$

where $\mathrm{t} 3 \in$ PRI $\left[\begin{array}{lllll}3 & 6 & 9 & 12 & \cdots\end{array}\right]$, and $\tau_{d}\left(t_{3} ; x, y\right)=\frac{2 R\left(t_{3} ; x, y\right)}{c}$. The echo of LOS 3 is squint forward-looking mode, and then it will be processed by the squint forward-looking SAR processing method.

After different LOS echoes are processed by independently, the multi-images will be received.

\section{Multi-images assembling}

After the processes above, images from different LOS angle are independently obtained. In order to get whole image of wide swath area, the multi-images assembling technology based on image registration (MIAT-IR) will be proposed in this article. This MIAT-IR includes two main steps.

First, image registration technology will be used in order to assemble the adjacent regions.

As Figure 5 shows, each two registration areas of the different adjacent LOS angle sub-images have the same size but different resolution. So, it is suitable for using correlation coefficient registration method. The correlation coefficient calculation formula is

$$
C=\frac{\sum\left[\left(I_{1}(x, y)-E\left(I_{1}(x, y)\right)\right)\right]\left[\left(I_{2}(x, y)-E\left(I_{2}(x, y)\right)\right)\right]}{\sqrt{\sum_{I_{1}}\left[\left(I_{1}(x, y)-E\left(I_{1}(x, y)\right)\right)\right]^{2}} \sqrt{\sum_{I_{2}}\left[\left(I_{2}(x, y)-E\left(I_{2}(x, y)\right)\right)\right]^{2}}}
$$




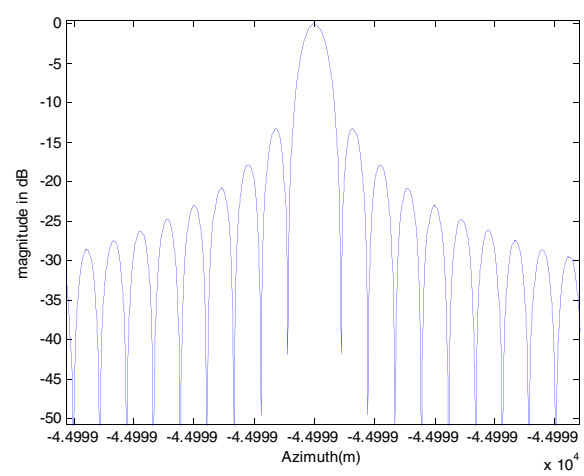

(a)

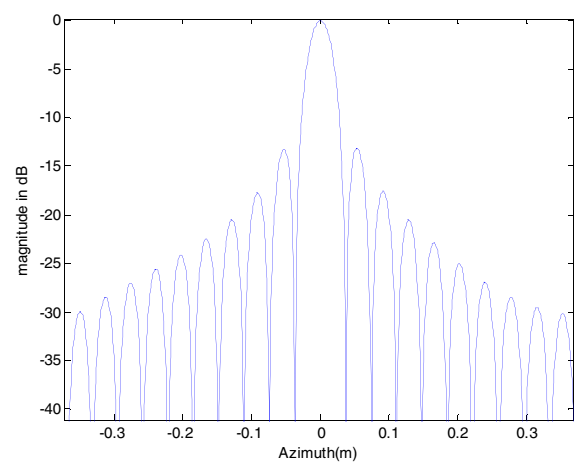

(b)

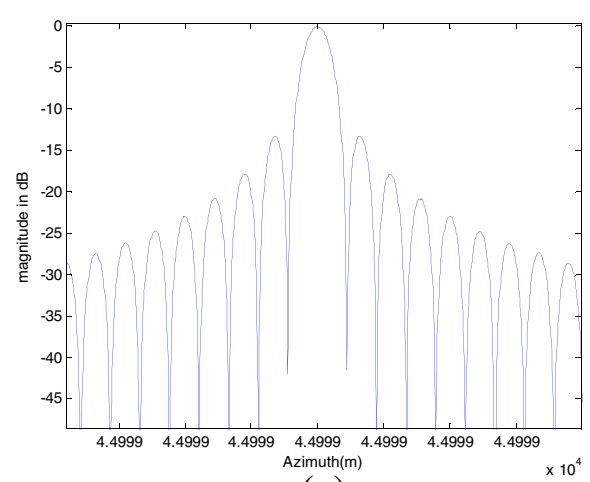

(c)

Figure 9 Azimuth profiles of targets A, O, and B. (a) Target A. (b) Target O. (c) Target B.

where $I_{1}(x, y)$ and $I_{2}(x, y)$ are the gray values of the two registration areas, and $E$ represents calculating mean value. The registration location is determined at the largest correlation location.

Second, remove the overlapping area that shares only part of whole aperture and assemble different parts together. Then the whole image of the large area has been obtained.

\section{Performance of an example system}

To evaluate the quantitative performance of NSLS-SAR HRWS and sustained imaging concepts, we consider an example system. The SAR operates in X-band with carrier frequency of $9.6 \mathrm{GHz}$. The detail corresponding system parameters are listed in Table 1.

In order to highlight the capacity of the proposed NSLS-SAR HRWS and sustained imaging technology, we set the target area distribution and it has been divided as shown in Figure 6. The number of joint aperture (viz. $N$ ) is 9 , and each aperture azimuth width is 10 $\mathrm{km}$. Targets A and B are the corner targets. Comparably, Target $\mathrm{O}$ locates at the center of the image area. Targets $\mathrm{C}$ and $\mathrm{D}$ are the two adjacent targets, which are belong to the adjacent two aperture areas, respectively. The imaging results of the five targets will be used to measure the effectiveness of the proposed NSLS-SAR HRWS and sustained imaging concepts.

The cost time to get the echo of the whole imaging area using the proposed JAT is $11 \mathrm{~min}$. While the required time of the traditional stripmap mode is $99 \mathrm{~min}$, which is nine times large as that of the proposed JAT in this article.

Figure 7 gives the difference between before and after the multi-image assembling. For detailed, Figure $7 \mathrm{a}$ is the azimuth profile of targets $C$ and $D$, which are the imaging result of LOS 7. Because in LOS 7, target D shares only part of whole aperture while target $C$ passes the whole aperture, then the azimuth resolution of target $\mathrm{D}$ is poor than that of target C. Comparably, Figure 7b has the opposite case. Lastly, Figure $7 \mathrm{c}$ is the azimuth profile of targets $C$ and $D$ that have been assembled. In this assembling process, we have used the MIAT-IR that is introduced in Section 4.2. Apparently, their resolutions or their image results of targets $C$ and $D$ are becoming the same. This figure verifies the effectiveness of the proposed multi-image assembling technology.

Figure 8 shows the contours of points A, O, and B operated by the proposed NSLS-SAR HRWS and sustained imaging technology. Figure 9 gives the azimuth profiles of the above three targets. In order to show the details, the results are interpolated by eight times. The 
resolution of targets $\mathrm{A}, \mathrm{O}$, and $\mathrm{B}$ are $0.68 \times 0.76 \mathrm{~m}^{2}$, $0.54 \times 0.75 \mathrm{~m}^{2}$, and $0.63 \times 0.77 \mathrm{~m}^{2}$. The peak sidelobe radio along azimuth directions of $\mathrm{A}, \mathrm{O}$, and $\mathrm{B}$ are $13.17,-13.39$, and $-13.28 \mathrm{~dB}$, respectively. The integrated sidelobe ratio along azimuth directions of $\mathrm{A}, \mathrm{O}$, and $\mathrm{B}$ are $-11.25,-11.46$, and $-11.36 \mathrm{~dB}$, respectively. This figure verifies that the proposed NSLS-SAR and the imaging strategy have the ability to achieve the HRWS imaging.

\section{Conclusion}

In order to achieve the HRWS, sustained imaging, and overcome the inherent defect of contradiction between azimuth high-resolution and rang wide swath for the high-speed platform, NSLS-SAR and its imaging strategy are proposed in this article. The first advantage of the proposed NSLS-SAR is that it can avoid the contradiction for high-speed platforms. At the same time, because of the low speed, it can also achieve the sustained imaging. While low-speed brings out the long synthetic time problem, then in order to deal with the slow imaging speed, the redundant PRF has been made full use of and the JAT is proposed to shorten the time needed for the large area imaging. Performance of the example system has verified the effectiveness of the proposed NSLS-SAR HRWS and sustained imaging concepts.

\section{Competing interest}

The authors declare that they have no competing interests.

\section{Acknowledgment}

The study was supported by the Pre-research Fund (no.

9140A07020412DZ02084) and the Pre-research Support (no. 62501023009).

Received: 12 December 2012 Accepted: 18 January 2013

Published: 4 March 2013

\section{References}

1. G Krieger, A Moreira, H Fiedler, I Hajnsek, M Werner, M Younis, M Zink, TanDEM-X: a satellite formation for high-resolution SAR interferometry IEEE Trans. Geosci. Remote Sens Pt. 1. 45(11), 3317-3341 (2007)

2. ZF Li, H Wang, T Su, Z Bao, Generation of wide-swath and high-resolution SAR images from multichannel small spaceborne SAR systems. IEEE Geosci Remote Sens Lett 2(1), 82-86 (2005)

3. EH Allen, The case for near-space. Aerosp Am 44(2), 31-34 (2006)

4. EB Tomme, The Paradigm Shift to Effects-Based Space: Near-Space as a Combat Space Effects Enabler, 2010. http://www.airpower.au.af.mil

5. W-Q Wang, Q Peng, J Cai, Digital beamforming for near-space wide-swath SAR imaging, in 8th International Symposium on Antennas, Propagation and EM Theory, 2008, 2008, pp. 1270-1273

6. W-Q Wang, Near-space wide-swath radar imaging with multiaperture antenna. IEEE Antenn. Wirel. Propagat. Lett 8, 461-464 (2009)

7. W-Q Wang, Q Peng, J Cai, Waveform-diversity-based millimeter-wave UAV SAR remote sensing. IEEE Trans. Geosci. Remote Sens 47(3), 691-700 (2009)

8. W-Q Wang, Near-space vehicle-borne SAR with reflector antenna for highresolution and wide-swath remote sensing. IEEE Trans. Geosci. Remote Sens 50(2), 338-348 (2012)

9. Z F Li, Z Bao, H Wang, G S Liao, Performance improvement for constellation SAR using signal processing techniques. IEEE Trans. Aerosp. Electron. Syst 42(2), 436-452 (2006)

10. A Jain, Multibeam synthetic aperture radar for global oceanography. IEEE Trans. Antenn. Propagat AP-27(4), 535-538 (1979)
11. BR Jean, JW Rouse, A multiple beam synthetic aperture radar design concept for geoscience applications. IEEE Trans. Geosci. Remote Sens GRS-21(2), 201-207 (1983)

12. EB Tomme, Balloons in today's military: an introduction to near-space concept. Air Space Power J 19(4), 39-50 (2005)

13. MJ Marcel, J Baker, Interdisciplinary design of a near-space vehicle, in Proceedings of Southeast Conference ( Richmond, VA, 2007), pp. 421-426

14. G David, M Mihael, Broadband Communications via High Altitude Platforms (Wiley, Hoboken, NJ, 2011)

15. A Currie, MA Brown, Wide-swath SAR. Proc. Inst. Elect. Eng. Radar Sonar Navigat 139(2), 122-135 (1992)

doi:10.1186/1687-1499-2013-57

Cite this article as: Yang et al:: Near-space slow SAR high-resolution wide swath and sustained imaging technology. EURASIP Journal on Wireless Communications and Networking 2013 2013:57.

\section{Submit your manuscript to a SpringerOpen ${ }^{\circ}$ journal and benefit from:}

- Convenient online submission

- Rigorous peer review

- Immediate publication on acceptance

- Open access: articles freely available online

- High visibility within the field

- Retaining the copyright to your article

Submit your next manuscript at springeropen.com 